\title{
PIECEWISE LINEAR DIFFERENTIAL SYSTEMS WITH ONLY CENTERS CAN CREATE LIMIT CYCLES?
}

\author{
JAUME LLIBRE ${ }^{1}$ AND MARCO ANTONIO TEIXEIRA ${ }^{2}$
}

\begin{abstract}
In this article we study the continuous and discontinuous planar piecewise differential systems formed only by linear centers separated by one or two parallel straight lines. When these piecewise differential systems are continuous they have no limit cycles. Also if they are discontinuous separated by a unique straight line they do not have limit cycles. But when the piecewise differential systems are discontinuous separated two parallel straight lines, we show that they can have at most one limit cycle, and that there exist such systems with one limit cycle.
\end{abstract}

\section{IntRoduCtion AND STATEMENT OF THE MAIN RESUlt}

A limit cycle is a periodic orbit of a differential system in $\mathbb{R}^{2}$ isolated in the set of all periodic orbits of that system. The study of the limit cycles goes back essentially to Poincaré [25] at the end of the 19th century. The existence of limit cycles became important in the applications to the real world, because many phenomena are related with their existence, see for instance the van der Pol oscillator [26, 27], or the Belousov-Zhabotinskii reaction which is a classical reaction of non-equilibrium thermodynamics appearing in a nonlinear chemical oscillator $[3,31], \ldots$

The study of the continuous piecewise linear differential systems separated by one or two parallel straight lines appear in a natural way in the control theory, see for instance the books $[2,10,12,13,19,24]$.

The easiest continuous piecewise linear differential systems are formed by two linear differential systems separated by a straight line. It is known that such systems have at most one limit cycle, see [7, 15, 21, 22]. But it is also known that if both linear differential systems are linear centers then the continuous piecewise linear differential system has no limit cycles.

Theorem 1. A continuous piecewise linear differential system separated by one straight line formed by two linear centers has no limit cycles.

2010 Mathematics Subject Classification. 34C29, 34C25, 47H11.

Key words and phrases. limit cycles, linear centers, continuous piecewise linear differential systems, discontinuous piecewise differential systems, first integrals. 
Since we cannot find an explicit reference where it is proved Theorem 1 and its proof is short and easy, we will provide it in section 2 .

Theorem 1 can be extended to continuous piecewise linear differential systems separated by two parallel straight lines formed by three linear centers.

Theorem 2. A continuous piecewise linear differential system separated by two parallel straight lines formed by three linear centers has no limit cycles.

Theorem 2 is proved in section 2 .

The study of the discontinuous piecewise linear differential systems separated by straight lines goes back to Andronov, Vitt and Khaikin [1] and until nowadays they had special attention from the mathematicians, mainly because these systems appear in mechanics, electrical circuits, economy, etc, see for instance the books [4, 29], and the the surveys [23, 30].

In the planar discontinuous piecewise linear differential systems the limit cycles can be of two kinds, sliding limit cycles or crossing limit cycles, the first ones contain some segment of the lines of discontinuity, and the second ones did not contain any of such segments. In this paper we only study the crossing limit cycles. In what follows when we talk about limit cycles we are talking about crossing limit cycles.

Again the easiest discontinuous piecewise linear differential systems are formed by two linear differential systems separated by a straight line. It is known that such systems can have three limit cycles, see $[5,6,9,11,14,16]$. It remains open to know if three is the maximum number of limit cycles that such systems can exhibit. In [18] we deal with discontinuous piecewise linear differential systems separated by a straight line such that each linear differential system has neither real nor virtual equilibria, and we show that such systems can have at most one limit cycle.

On the other hand, in [20] it is proved:

Theorem 3. A discontinuous piecewise linear differential system separated by one straight line formed by two linear centers has no limit cycles.

Since the proof of Theorem 3 is short we shall include it in section 3 for completeness. We remark that the problem studied in Theorem 3 but changing the linear centers by linear foci becomes a difficult problem, already studied by several authors, see in particular the paper [8] and the references quoted therein.

Here we shall prove that Theorem 3 cannot be extended to discontinuous piecewise linear differential system separated by two parallel straight lines formed by three linear centers. Thus our main result is:

Theorem 4. A discontinuous piecewise linear differential system separated by two parallel straight lines formed by three linear centers can have at most 
one limit cycle. Moreover there are systems in this class having one limit cycle.

Theorem 4 is proved in section 3 .

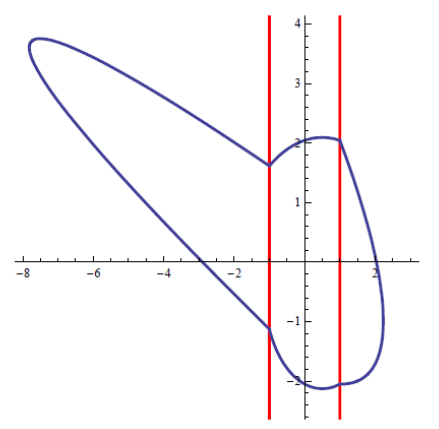

FiguRE 1. The limit cycle of the discontinuous piecewise linear differential system (1).

Consider the discontinuous piecewise linear differential system separated by the two parallel straight lines $x= \pm 1$ defined by$$
\dot{x}=(16+32 x+65 y) / 2, \quad \dot{y}=-8(x+2 y), \quad \text { if } x<-1 .
$$

$$
\begin{array}{lll}
\dot{x}=2(-4+4 x+5 y), & \dot{y}=-8(1+x+y), & \text { if } x>1, \\
\dot{x}=1+2 y, & \dot{y}=1-2 x, & \text { if }-1<x<1,
\end{array}
$$

This system has the limit cycle of Figure 1. This will be proved in section 3 .

We remark that it is not difficult to show that if a discontinuous piecewise differential system with two straight lines of discontinuity is symmetric with respect to the origin of coordinates, then when this system has a periodic solution it has a continuum of periodic solutions. So the lack of symmetry plays a role for the existence of limit cycles for the systems studied in Theorem 4 .

\section{Preliminaries And the proof of Theorems 1 And 2}

The following lemma provides a normal form for an arbitrary linear differential system having a center.

Lemma 5. A linear differential system having a center can be written as

$$
\dot{x}=-b x-\frac{4 b^{2}+\omega^{2}}{4 a} y+d, \quad \dot{y}=a x+b y+c,
$$

with $a>0$ and $\omega>0$. 
Proof. Consider a general linear differential system

$$
\dot{x}=A x+B y+d, \quad \dot{y}=a x+b y+c,
$$

in the $\mathbb{R}^{2}$ and assume that it has a center. The eigenvalues of this system are

$$
\frac{A+b \pm \sqrt{4 a B+(A-b)^{2}}}{2} .
$$

Then this system has a center if $A+b=0$ and $4 a B+(A-b)^{2}=-\omega^{2}$ for some $\omega>0$ and $a B<0$, i.e. if $A=-b, B=-\left(4 b^{2}+\omega^{2}\right) /(4 a)$ and $a>0$. Hence the lemma is proved.

Proof of Theorem 1. Assume that we have a continuous piecewise linear differential system separated by one straight line and formed by two centers. Without loss of generality we can assume that the straight line of continuity is $x=0$. Doing a linear change of coordinates we can write the matrix of the linear center in $x<0$ in its real Jordan normal form, so the system in $x<0$ becomes

$$
\dot{x}=-y-\beta, \quad \dot{y}=-x+\gamma,
$$

and it has the first integral

$$
H_{2}(x, y)=(y+\beta)^{2}+(x-\gamma)^{2} .
$$

After this linear change of variables the center in $x>0$ has a general expression as the (2) given in the Lemma 5 . Note that system (2) has the first integral

$$
H_{1}(x, y)=4(a x+b y)^{2}+8 a(c x-d y)+y^{2} \omega^{2} .
$$

Since we must have a continuous piecewise linear differential system both systems, the (2) and the (3) must coincide on $x=0$, therefore

$$
a=\omega^{2} / 4, \quad b=0, \quad c=-\gamma, \quad d=-\beta .
$$

Clearly if the continuous piecewise linear differential system has a periodic orbit candidate to be a limit cycle, because its two differential systems are linear centers and consequently their orbits are ellipses, such a periodic orbit must intersect the line $x=0$ in exactly two points, namely $\left(0, y_{1}\right)$ and $\left(0, y_{2}\right)$ with $y_{1}<y_{2}$. Since $H_{1}$ and $H_{2}$ are two first integrals we have that

$$
H_{1}\left(0, y_{1}\right)-H_{1}\left(0, y_{2}\right)=0, \quad \text { and } H_{2}\left(0, y_{1}\right)-H_{2}\left(0, y_{2}\right)=0 \text {. }
$$

That is

$$
\begin{aligned}
& \left(y_{1}-y_{2}\right)\left(2 \beta+y_{1}+y_{2}\right) \omega^{2}=0 \\
& \left(y_{1}-y_{2}\right)\left(2 \beta+y_{1}+y_{2}\right)=0 .
\end{aligned}
$$

The solutions $\left(y_{1}, y_{2}\right)$ of this last system satisfying the necessary condition $y_{1}<y_{2}$ are $y_{2}=-2 \beta-y_{1}$ with $y_{1}$ arbitrary. So the periodic orbits of the continuous piecewise linear differential system are in a continuum of periodic 
orbits, and consequently this differential system has no limit cycles. This completes the proof of the theorem.

Proof of Theorem 2. Suppose that we have a continuous piecewise linear differential system separated by two parallel straight lines formed by three linear centers. Doing a rotation and a scaling of the variables we can assume without loss of generality that the two parallel straight lines of continuity are $x= \pm 1$. As in the proof of Theorem 1 we can assume that the linear center in the strip $-1<x<1$ is given by the system (3), that the linear center in the half-plane $x>1$ is given by the system (2), and finally from Lemma 5 that the linear center in the half-plane $x<-1$ can be given by the system

$$
\dot{x}=-B x-\frac{4 B^{2}+\Omega^{2}}{4 A} y+D, \quad \dot{y}=A x+B y+C,
$$

with $A>0, \Omega>0$ and having the first integral

$$
H_{3}(x, y)=4(A x+B y)^{2}+8 A(C x-D y)+y^{2} \Omega^{2} .
$$

Since we have a continuous piecewise linear differential system it follows that system (2) and (3) must coincide on the straight line $x=1$, and system (3) and (5) must coincide on the straight line $x=-1$. So we obtain that

$$
a=\left(4+\omega^{2}\right) / 4, \quad b=1, \quad c=-\left(4+4 \gamma+\omega^{2}\right) / 4, \quad d=1-\beta,
$$

and

$$
A=\left(4+\Omega^{2}\right) / 4, \quad B=1, \quad C=\left(4-4 \gamma+\Omega^{2}\right) / 4, \quad D=-1-\beta,
$$

respectively.

By Theorem 1 if this continuous piecewise linear differential system has a limit cycle this must intersect the three open regions $x<-1,-1<x<1$ and $x>1$. Since the orbits in each one of these three regions are ellipses or a piece of one ellipse, a possible limit cycle must intersect each straight line $x= \pm 1$ in exactly two points, namely $\left(1, y_{1}\right),\left(1, y_{2}\right),\left(-1, y_{3}\right)$ and $\left(-1, y_{4}\right)$ with $y_{1}<y_{2}$ and $y_{3}>y_{4}$. Hence the first integrals $H_{1}, H_{2}$ and $H_{3}$ must satisfy

$$
\begin{aligned}
& H_{1}\left(1, y_{1}\right)-H_{1}\left(1, y_{2}\right)=0 \\
& H_{2}\left(1, y_{2}\right)-H_{2}\left(-1, y_{3}\right)=0 \\
& H_{3}\left(-1, y_{3}\right)-H_{3}\left(-1, y_{4}\right)=0 \\
& H_{2}\left(-1, y_{4}\right)-H_{2}\left(1, y_{1}\right)=0
\end{aligned}
$$


or equivalently

$$
\begin{aligned}
& \left(y_{1}-y_{2}\right)\left(y_{1}+y_{2}+2 \beta\right)\left(4+\omega^{2}\right)=0, \\
& y_{2}^{2}-y_{3}^{2}+2 \beta\left(y_{2}-y_{3}\right)-4 \gamma=0, \\
& \left(y_{3}-y_{4}\right)\left(y_{3}+y_{4}+2 \beta\right)\left(4+\Omega^{2}\right)=0, \\
& y_{1}^{2}-y_{4}^{2}+2 \beta\left(y_{1}-y_{4}\right)-4 \gamma=0,
\end{aligned}
$$

The solutions $\left(y_{1}, y_{2}, y_{3}, y_{4}\right)$ of this last system satisfying the necessary conditions $y_{1}<y_{2}$ and $y_{3}>y_{4}$ are

$$
\left(-\beta-\sqrt{y_{4}^{2}+2 \beta y_{4}+\beta^{2}+4 \gamma}, \sqrt{y_{4}^{2}+2 \beta y_{4}+\beta^{2}+4 \gamma}-\beta,-y_{4}-2 \beta, y_{4}\right),
$$

with $y_{4}$ arbitrary. So the periodic orbits of the continuous piecewise linear differential system are in a continuum of periodic orbits, and consequently this differential system has no limit cycles. This completes the proof of the theorem.

\section{Proof of Theorems 3 And 4}

Proof of Theorem 3. Assume that we have a discontinuous piecewise linear differential system separated by one straight line and formed by two centers. As in the proof of Theorem 1 we can suppose that the straight line of discontinuity is $x=0$, and that the linear center in the half-plane $x<0$ is given by the system (3), while the linear center in the half-plane $x>0$ is given by the system (2). Therefore if the piecewise linear differential system has a periodic orbit candidate to be a limit cycle it must intersect the line $x=0$ in exactly two points, namely $\left(0, y_{1}\right)$ and $\left(0, y_{2}\right)$ with $y_{1}<y_{2}$. Since $H_{1}$ and $H_{2}$ are two first integrals they must satisfy the system (4), which now is equivalent to the system

$$
\begin{aligned}
& \left(y_{1}-y_{2}\right)\left(-8 a d+\left(4 b^{2}+\omega^{2}\right)\left(y_{1}+y_{2}\right)\right)=0, \\
& \left(y_{1}-y_{2}\right)\left(2 \beta+y_{1}+y_{2}\right)=0 .
\end{aligned}
$$

This system has either no solutions $\left(y_{1}, y_{2}\right)$ satisfying the necessary condition $y_{1}<y_{2}$, or it has a continuum of solutions. So the continuous piecewise linear differential system either does not have periodic solutions, or it has a continuum of periodic orbits, and consequently this differential system has no limit cycles. This completes the proof of the theorem.

Proof of Theorem 4. Assume that we have a discontinuous piecewise linear differential system separated by two parallel straight lines formed by three linear centers. As in the proof of Theorem 2 we can suppose that the two discontinuous parallel straight lines are $x= \pm 1$, and that the systems having a linear center are (2) in $x>1,(3)$ in $-1<x<1$, and (5) in $x<-1$.

By the same arguments than in the proof of Theorem 2 a possible limit cycle of this discontinuous piecewise linear differential system must intersect 
each discontinuous straight line in two points, namely $\left(1, y_{1}\right),\left(1, y_{2}\right),\left(-1, y_{3}\right)$ and $\left(-1, y_{4}\right)$ with $y_{1}<y_{2}$ and $y_{3}>y_{4}$, and the first integrals $H_{1}, H_{2}$ and $\mathrm{H}_{3}$ must satisfy the four equations (6), which now for our discontinuous piecewise linear differential system are equal to

$$
\begin{aligned}
& \left(y_{1}-y_{2}\right)\left(8 a(b-d)+\left(4 b^{2}+\omega^{2}\right)\left(y_{1}+y_{2}\right)\right)=0, \\
& y_{2}^{2}-y_{3}^{2}+2 \beta\left(y_{2}-y_{3}\right)-4 \gamma=0, \\
& \left(y_{3}-y_{4}\right)\left(8 A(B+D)-\left(4 B^{2}+\Omega^{2}\right)\left(y_{3}+y_{4}\right)\right)=0, \\
& y_{4}^{2}-y_{1}^{2}+2 \beta\left(y_{4}-y_{1}\right)+4 \gamma=0 .
\end{aligned}
$$

Since $y_{1}<y_{2}$ and $y_{3}>y_{4}$ the previous system is equivalent to the system

$$
\begin{aligned}
& r+k\left(y_{1}+y_{2}\right)=0, \\
& \left(y_{2}-y_{3}\right)\left(y_{2}+y_{3}+2 \beta\right)-\Gamma=0, \\
& R+K\left(y_{3}+y_{4}\right)=0 \\
& \left(y_{1}-y_{4}\right)\left(y_{1}+y_{4}+2 \beta\right)+\Gamma=0,
\end{aligned}
$$

where

$r=8 a(b-d), \quad k=\left(4 b^{2}+\omega^{2}\right), \quad \Gamma=4 \gamma, \quad R=8 A(B+D), \quad K=-\left(4 B^{2}+\Omega^{2}\right)$.

Looking at system (7) we remark that if $\left(y_{1}, y_{2}, y_{3}, y_{4}\right)$ is a solution, then $\left(y_{2}, y_{1}, y_{4}, y_{3}\right)$ is also a solution, but due to the fact that $y_{1}<y_{2}$ and $y_{3}>y_{4}$, at most one of these two solutions will be satisfactory.

Since $K \neq 0$ from the third equation of (7) we isolated $y_{3}$ and substitute it in the second equation of (7), and we get

(8) $2 K R \beta-R^{2}-K^{2} \Gamma+2 K^{2} \beta y_{2}+2 K(K \beta-R) y_{4}+K^{2}\left(y_{2}^{2}-y_{4}^{2}\right)=0$.

In a similar way since $k \neq 0$ from the first equation of $(7)$ we isolated $y_{1}$ and substitute it in the fourth equation of (7), and we obtain

$$
r^{2}-2 k r \beta+k^{2} \Gamma-2 k y_{2}(k \beta-r)-2 k^{2} \beta y_{4}+k^{2}\left(y_{2}^{2}-y_{4}^{2}\right)=0 .
$$

Now doing the Gröbner basis of the two left hand polynomials in the equations (8) and (9) with respect the variables $y_{2}$ and $y_{4}$, we obtain the two equations

$$
\begin{aligned}
& a_{0}+a_{1} y_{4}+a_{2} y_{4}^{2}=0, \\
& b_{0}+b_{1} y_{2}+b_{2} y_{4}=0,
\end{aligned}
$$


where

$$
\begin{aligned}
a_{0}= & R^{4} k^{4}+16 K^{3} R \beta^{3} k^{4}-4 K^{2} R^{2} \beta^{2} k^{4}+4 K^{4} \Gamma^{2} k^{4}-4 K R^{3} \beta k^{4} \\
& +4 K^{2} R^{2} \Gamma k^{4}-8 K^{3} R \beta \Gamma k^{4}-16 K^{4} r \beta^{3} k^{3}-16 K^{3} r R \beta^{2} k^{3} \\
& +8 K^{2} r R^{2} \beta k^{3}-2 K^{2} r^{2} R^{2} k^{2}+20 K^{4} r^{2} \beta^{2} k^{2}+4 K^{3} r^{2} R \beta k^{2} \\
& -8 K^{4} r^{3} \beta k+K^{4} r^{4}, \\
a_{1}= & -4 k^{2} K\left(4 k^{2} \beta \Gamma K^{3}+r^{2} R K^{2}-4 k r R \beta K^{2}-2 k^{2} R \Gamma K^{2}\right. \\
& \left.+4 k^{2} R^{2} \beta K-k^{2} R^{3}\right), \\
a_{2}= & -4 k^{2} K^{2}(k R-K r)(-K r-k R+4 k K \beta), \\
b_{0}= & -R^{2} k^{2}+2 K R \beta k^{2}-2 K^{2} \Gamma k^{2}+2 K^{2} r \beta k-K^{2} r^{2}, \\
b_{1}= & 2 k K^{2}(2 k \beta-r), \\
b_{2}= & 2 k^{2} K(2 K \beta-R) .
\end{aligned}
$$

Eventually system (10) could have a continuum of solutions $\left(y_{2}, y_{4}\right)$ if some coefficients of the polynomials that there appear are zero, but then the possible periodic solutions would not be limit cycles. So assume that system (10) has finitely many solutions. We recall that Bezout Theorem (see for instance [28]) states that if a polynomial differential system of equations has finitely many solutions, then the number of its solutions is at most the product of the degrees of the polynomials which appear in the system. Then by Bezout Theorem system (7) has at most two solutions. Finally from these at most two solutions $\left(y_{2}, y_{4}\right)$ of system (10) we get two solutions $\left(y_{1}, y_{2}, y_{3}, y_{4}\right)$ of system (7), but from the previous remark at most one of these two solution would satisfy $y_{1}<y_{2}$ and $y_{3}>y_{4}$. In summary, we have proved that at most we can have one limit cycle.

Now we shall prove that the discontinuous piecewise linear differential system (1) having a center in each of these three pieces has also one limit cycle. This will complete the proof of Theorem 1.

The eigenvalues of the matrices of the three linear differential systems (1) are $\pm 4 i, \pm 2 i$ and $\pm 2 i$, respectively. So the three systems have a linear center.

The first integrals of the three linear differential systems (1) are

$$
\begin{aligned}
& H_{1}(x, y)=8 x+4 x^{2}-8 y+8 x y+5 y^{2} \\
& H_{2}(x, y)=y+y^{2}-x+x^{2} \\
& H_{3}(x, y)=8 y+\frac{1}{4} y^{2}+4(x+2 y)^{2}
\end{aligned}
$$


respectively. Then system (6) for the discontinuous piecewise linear differential system (1) becomes

$$
\begin{aligned}
& 5\left(y_{1}-y_{2}\right)\left(y_{1}+y_{2}\right)=0, \\
& -2+y_{2}+y_{2}^{2}-y_{3}-y_{3}^{2}=0, \\
& \frac{1}{4}\left(y_{3}-y_{4}\right)\left(-32+65 y_{3}+65 y_{4}\right)=0, \\
& 2-y_{1}-y_{1}^{2}+y_{4}+y_{4}^{2}=0 .
\end{aligned}
$$

Taking into account that $y_{1}<y_{2}$ and $y_{3}>y_{4}$ the unique solution $\left(y_{1}^{*}, y_{2}^{*}, y_{3}^{*}, y_{4}^{*}\right)$ of the previous system is

$$
\left(-\frac{97}{2340} \sqrt{\frac{4873}{2}}, \frac{97}{2340} \sqrt{\frac{4873}{2}}, \frac{16}{65}+\frac{1}{36} \sqrt{\frac{4873}{2}}, \frac{16}{65}-\frac{1}{36} \sqrt{\frac{4873}{2}}\right) .
$$

The solution $\left(x_{1}(t), y_{1}(t)\right)$ of the first linear differential system of (1) such that $\left(x_{1}(0), y_{1}(0)\right)=\left(1, y_{1}^{*}\right)$ is

$$
\begin{aligned}
& x_{1}(t)=10 \cos (4 t)+\frac{97}{936} \sqrt{\frac{4873}{2}} \sin (4 t)-9 \\
& y_{1}(t)=\left(-8+\frac{97}{2340} \sqrt{\frac{4873}{2}}\right) \cos (4 t)-\left(4+\frac{97}{1170} \sqrt{\frac{4873}{2}}\right) \sin (4 t)+8 .
\end{aligned}
$$

The solution $\left(x_{2}(t), y_{2}(t)\right)$ of the second linear differential system of (1) such that $\left(x_{2}(0), y_{2}(0)\right)=\left(1, y_{2}^{*}\right)$ is

$$
\begin{aligned}
& x_{2}(t)=\frac{2340}{4680}(\cos (2 t)+(2340-97 \sqrt{9746}) \sin (2 t)+2340), \\
& y_{2}(t)=\frac{1}{4680}((2340-97 \sqrt{9746}) \cos (2 t)-2340(\sin (2 t)+1)) .
\end{aligned}
$$

The solution $\left(x_{1}(t), y_{1}(t)\right)$ of the third linear differential system of (1) such that $\left(x_{3}(0), y_{3}(0)\right)=\left(-1, y_{3}^{*}\right)$ is

$$
\begin{aligned}
& x_{3}(t)=-33 \cos (2 t)-\frac{65}{144} \sqrt{\frac{4873}{2}} \sin (2 t)+32, \\
& y_{3}(t)=\frac{(76032-65 \sqrt{9746})}{4680} \cos (2 t)+\left(\frac{132}{65}+\frac{\sqrt{9746}}{9}\right) \sin (2 t)-16 .
\end{aligned}
$$

The solution $\left(x_{4}(t), y_{4}(t)\right)$ of the second linear differential system of (1) such that $\left(x_{4}(0), y_{4}(0)\right)=\left(-1, y_{4}^{*}\right)$ is

$$
\begin{aligned}
& x_{4}(t)=-\frac{3}{2} \cos (2 t)+\frac{(3492+65 \sqrt{9746})}{4680} \sin (2 t)+\frac{1}{2}, \\
& y_{4}(t)=\frac{(3492+65 \sqrt{9746})}{4680} \cos (2 t)+\frac{3}{2} \sin (2 t)-\frac{1}{2} .
\end{aligned}
$$


The time that the solution $\left(x_{1}(t), y_{1}(t)\right)$ contained in $x>1$ needs to reach the point $\left(1, y_{2}^{*}\right)$ is $t_{1}=0.2364182295331372$. . The time that the solution $\left(x_{2}(t), y_{2}(t)\right)$ contained in $-1<x<1$ needs to reach the point $\left(-1, y_{3}^{*}\right)$ is $t_{2}=0.7443915397542379$. The time that the solution $\left(x_{3}(t), y_{3}(t)\right)$ contained in $x<-1$ needs to reach the point $\left(-1, y_{4}^{*}\right)$ is $t_{3}=0.593873916108556$. Finally the time that the solution $\left(x_{4}(t), y_{4}(t)\right)$ contained in $-1<x<1$ needs to reach the point $\left(1, y_{1}^{*}\right)$ is $t_{4}=0.405141655169058$..

Drawing the orbits $\left(x_{k}(t), y_{k}(t)\right)$ for the times $t \in\left[0, t_{k}\right]$ we obtain the limit cycle of Figure 1.

\section{Concluding SeCtion}

We have studied the continuous and discontinuous planar piecewise differential systems formed only by linear centers separated by one or two parallel straight lines.

Such continuous piecewise differential systems appear in control theory, while the discontinuous ones appear in mechanics, electrical circuits, economy, etc.

When these piecewise differential systems are continuous or discontinuous separated by a unique straight line we prove that they have no limit cycles. But when the piecewise differential systems are discontinuous separated two parallel straight lines, we show that they can have at most one limit cycle, and that there exist such systems with one limit cycle.

\section{ACKNOWLEDGMENTS}

We thank to the reviewers their comments which help us to improve this paper.

The first author is partially supported by a FEDER-MINECO grant MTM2016-77278-P, a MINECO grant MTM2013-40998-P, an AGAUR grant number 2014SGR-568, and a CAPES grant 88881. 030454/ 2013-01 do Programa CSF-PVE. The second author is partially supported by FAPESP under grant number 2012/18780-0.

\section{REFERENCES}

[1] A. Andronov, A. Vitt and S. Khaikin, Theory of Oscillations, Pergamon Press, Oxford, 1966.

[2] D.P. Atherton, Nonlinear control engineering, Van Nostrand Reinhold Co. Ltd., New York, 1982.

[3] B.P. Belousov, Periodically acting reaction and its mechanism, Collection of abstracts on radiation medicine, Moscow, 1958, pp. 145-147. 
[4] M. di Bernardo, C. J. Budd, A. R. Champneys and P. Kowalczyk, PiecewiseSmooth Dynamical Systems: Theory and Applications, Appl. Math. Sci. Series 163, Springer-Verlag, London, 2008.

[5] D.C. Braga AND L.F. Mello, Limit cycles in a family of discontinuous piecewise linear differential systems with two zones in the plane, Nonlinear Dynam. 73 (2013), $1283-1288$.

[6] C. Buzzi, C. Pessoa And J. Torregrosa, Piecewise linear perturbations of a linear center, Discrete Contin. Dyn. Syst. 9 (2013), 3915-3936.

[7] E. Freire, E. Ponce, F. Rodrigo and F. Torres, Bifurcation sets of continuous piecewise linear systems with two zones, Internat. J. Bifur. Chaos 8 (1998), 2073-2097.

[8] E. Freire, E. Ponce and F. Torres, Canonical Discontinuous Planar Piecewise Linear Systems, SIAM J. Applied Dynamical Systems 11, (2012), 181-211.

[9] E. Freire, E. Ponce And F. Torres, A general mechanism to generate three limit cycles in planar Filippov systems with two zones, Nonlinear Dynam. 78 (2014), 251263.

[10] M.A. Henson And D.E. Seborg, Nonlinear process control, Prentice-Hall, New Jersey, 1997.

[11] S.M. HuAN AND X.S. YANG, On the number of limit cycles in general planar piecewise linear systems, Discrete Contin. Dynam. Syst. Ser. A 32 (2012), 2147-2164.

[12] A. Isidori, Nonlinear control systems, Springer-Verlag, London, 1996.

[13] O. Katsuhiko, Modern Control Engineering, 2nd ed., Prentice-Hall, USA, 1990.

[14] L. LI, Three crossing limit cycles in planar piecewise linear systems with saddle-focus type, Electron. J. Qual. Theory Differ. Equ. 2014, no. 70, pp. 14.

[15] J. Llibre, M. OrdóñEz And E. Ponce, On the existence and uniqueness of limit cycles in a planar piecewise linear systems without symmetry, Nonlinear Anal. Ser. B: Real World Appl. 14 (2013), 2002-2012.

[16] J. Llibre And E. Ponce, Three nested limit cycles in discontinuous piecewise linear differential systems with two zones, Dynam. Contin. Discrete Impul. Syst. Ser. B 19 (2012), 325-335.

[17] J. Llibre And J. Sotomayor, Phase portraits of planar control systems, Nonlinear Analysis, Theory, Methods and Applications 27 (1996), 1177-1197.

[18] J. Llibre AND M.A. TeIXeIRA, Piecewise linear differential systems without equilibria produce limit cycles? to appear in Nonlinear Dynamics (2017), doi:10.1007/s11071-016-3236-9.

[19] J. Llibre And A. Teruel, Introduction to the Qualitative Theory of Differential Systems, Planar, Symmetric and Continuous Piecewise Linear Differential Systems, Birkhauser Advanced Texts, 2014.

[20] J. Llibre AND X. ZhANG, Limit cycles for discontinuous planar piecewise linear differential systems, preprint, 2016.

[21] R. Lum, L.O. ChuA, Global properties of continuous piecewise-linear vector fields. Part I: Simplest case in $\mathbb{R}^{2}$, Internat. J. Circuit Theory Appl. 19 (1991), 251-307.

[22] R. Lum AND L.O. ChuA, Global properties of continuous piecewise-linear vector fields. Part II: simplest symmetric in $\mathbb{R}^{2}$, Internat. J. Circuit Theory Appl. 20 (1992), $9-46$.

[23] O. Makarenkov and J.S.W. Lamb, Dynamics and bifurcations of nonsmooth systems: A survey, Physica D 241 (2012), 1826-1844.

[24] S. NARENDRA AND J. M. TAYLOR, Frequency domain criteria for absolute stability, Acad. Press, New York, 1973.

[25] H. Poincaré, Sur l'intégration des équations différentielles du premier ordre et du premier degré I and II, Rendiconti del Circolo Matematico di Palermo 5 (1891), 161-191; 11 (1897), 193-239.

[26] B. VAN DER POL, A theory of the amplitude of free and forced triode vibrations, Radio Review (later Wireless World) 1 (1920), 701-710. 
[27] B. VAn DeR Pol, On relaxation-oscillations, The London, Edinburgh and Dublin Phil. Mag. \& J. of Sci. 2(7) (1926), 978-992.

[28] I.R. Shafarevich, Basic Algebraic Geometry, Springer, 1974.

[29] D.J.W. Simpson, Bifurcations in Piecewise-Smooth Continuous Systems, World Scientific Series on Nonlinear Science A, vol 69, World Scientific, Singapore, 2010.

[30] M.A. Teixeira, Perturbation theory for non-smooth systems, In Mathematics of complexity and dynamical systems. Vols. 1-3, Springer, New York, 2012, pp. 13251336.

[31] A.M. Zhabotinsky, Periodical oxidation of malonic acid in solution (a study of the Belousov reaction kinetics), Biofizika 9 (1964), 306-311.

${ }^{1}$ Departament de Matemàtiques, Universitat Autònoma de Barcelona, 08193 Bellaterra, Barcelona, Catalonia, Spain

E-mail address: jllibre@mat.uab.cat

2 Departamento de Matemática, Universidade Estadual de Campinas, Caixa Postal 6065, 13083-970, Campinas, SP, Brazil

E-mail address: teixeira@ime.unicamp.br 